\title{
EDITORIAL
}

\section{Nordic STS transition}

By Tomas Moe Skjølsvold

Two years ago, I visited Cornell University, who hosted an event to celebrate that 40 years had passed since they hosted the first $4 \mathrm{~S}$ conference in a small room at campus. Since then, the annual $4 \mathrm{~S}$ meeting has evolved into an academic mega event with thousands of delegates from around the world. This year, celebrations occur closer to home, as the STS Centre at NTNU, Trondheim reaches the age of 30 . Thus, here too, STS has become a force to be reckoned with, as there are multiple STS centers in all Nordic countries, regular conferences, several journals and countless scholars who work under the banner within many disciplines. Hence, Nordic STS is growing up.

This moment, resembling a rite-de-passage, would be a nice opportunity to engage in retrospective appraisal of achievements. While this would be warranted, I find it more fruitful simply to acknowledge the tremendous work of the generations before us. They have built a solid body of theoretical and empirical scholarship and trained countless students. Just as important, they have paved the way for many careers in STS and related fields, through opening up a series of institutional spaces in Nordic universities and other research institutions, where STS today is a legitimate and important field in its own right. Such stable institutional anchoring has been essential, because it has allowed for collective efforts of experimentation with regards scholarly practices, public engagement, and in the case of the Nordic Journal of Science and Technology Studies, publication practices.

The current issue of NJSTS represents an effort to continue this experimentation. As usual, we have a nice selection of peer reviewed research articles, which addresses key concerns within and around contemporary techno-science. The topics this time ranges from digitalization to welfare technology and asking how Science talents become what they are. In addition, there is artwork and a book review and we do hope you find this eclectic collection of to be of interest.

In other news, NJSTS is also in the midst of a transition, as this is my final editorial note. In fact, this issue has been co-edited together with a new editorial duo consisting of Roger Andre Søraa and Jenny Melind Bergschöld (Read more about Roger here: https://www.ntnu.edu/employees/roger.soraa and Jenny here: https://www.ntnu.edu/employees/jenny.bergschold). I am confident that this transition in NJSTS will prove successful and that the journal under their management will become an even more exciting venue for Nordic STS research than it has been in the past.

Editing NJSTS has been a great privilege. The community of reviewers has been overwhelmingly positive, rejecting requests only in rare occasions. This is tremendously important for the journal, and I do hope you all keep this spirit up, despite being busy with everyday academic life. It has been great to observe the diversity of Nordic STS work up close, and it is nice to see that the field is thriving. NJSTS has an important role to play over the next years in keeping this momentum up.

Thus, I wish the new editors all the best, and I really look forward to following the journal further as an interested reader. 